\title{
A Transaction Trade-Off Utility Function Approach for Predicting the End-Price of Online Auctions in IoT
}

\author{
Xiaohui Li $\mathbb{D D}^{1,2}$ and Hongbin Dong $\mathbb{D}^{1}$ \\ ${ }^{1}$ College of Computer Science and Technology, Harbin Engineering University, Harbin 150001, China \\ ${ }^{2}$ Harbin Vocational \& Technical College, Harbin 150081, China \\ Correspondence should be addressed to Hongbin Dong; donghongbin@hrbeu.edu.cn
}

Received 9 November 2020; Revised 11 December 2020; Accepted 23 January 2021; Published 3 February 2021

Academic Editor: Yaguang Lin

Copyright (c) 2021 Xiaohui Li and Hongbin Dong. This is an open access article distributed under the Creative Commons Attribution License, which permits unrestricted use, distribution, and reproduction in any medium, provided the original work is properly cited.

\begin{abstract}
To stimulate large-scale users to participate in the big data construction of IoT (internet of things), auction mechanisms based on game theory are used to select participants and calculate the corresponding reward in the process of crowdsensing data collection from IoT. In online auctions, bidders bid many times and increase their bid price. All the bidders want to maximize their utility in auctions. An effective incentive mechanism can maximize social welfare in online auctions. It is complicated for auction platforms to calculate social welfare and the utility of each bidder's bidding items in online auctions. In this paper, a transaction trade-off utility incentive mechanism is introduced. Based on the transaction trade-off utility incentive mechanism, it can make the forecasting process consistent with bidding behaviors. Furthermore, an end-price dynamic forecasting agent is proposed for predicting end prices of online auctions. The agent develops a novel trade-off methodology for classifying online auctions by using the transaction trade-off utility function to measure the distance of auction items in KNN. Then, it predicts the end prices of online auctions by regression. The experimental results demonstrate that an online auction process considering the transaction utility is more consistent with the behaviors of bidders, and the proposed prediction algorithm can obtain higher prediction accuracy.
\end{abstract}

\section{Introduction}

With the rapid development of IoT and e-commerce, the traditional model of commodity trading and resource allocation has changed. Online market platforms like eBay, Yahoo, and Amazon have attracted more and more trading users. eBay is the leading auction market platform, and it adopts the English auction format. There are more than 100 million members and 20 million items for sale at any given time. Auction is an important mechanism of economic exchange [1]. Online auction is an online marketing model on the internet, which has turned out to be an effective way to allocate goods and resources [2-4]. It has become an important form of e-commerce. Online auctions have attracted more and more scholars' attention and research. Online auctions will produce a large amount of electronic transaction data in a transaction process, which contains enough economic behavior information and product information. A lot of researchers studied the distributed data collection and privacy problems [5-7]. It is beneficial for all buyers, sellers, and marketplace managers to make full use of these transaction data for predicting the end prices of online auctions using machine learning algorithms, data mining technology, and time series analysis [8-10].

Many firms can be offered a great benefit by efficient strategies in social networks [11]. An auction problem can be regarded as a resource allocation problem [12-14]. To allocate resources reasonably, the utility should be considered. Considering transaction utility is more suitable for bidding behaviors in auctions. As the utility of items is different for everyone in online auctions, not all items can be sold at a uniform price. We restrict items to bidders with very simple utility functions which we call "transaction trade-off utility function" in this paper. 


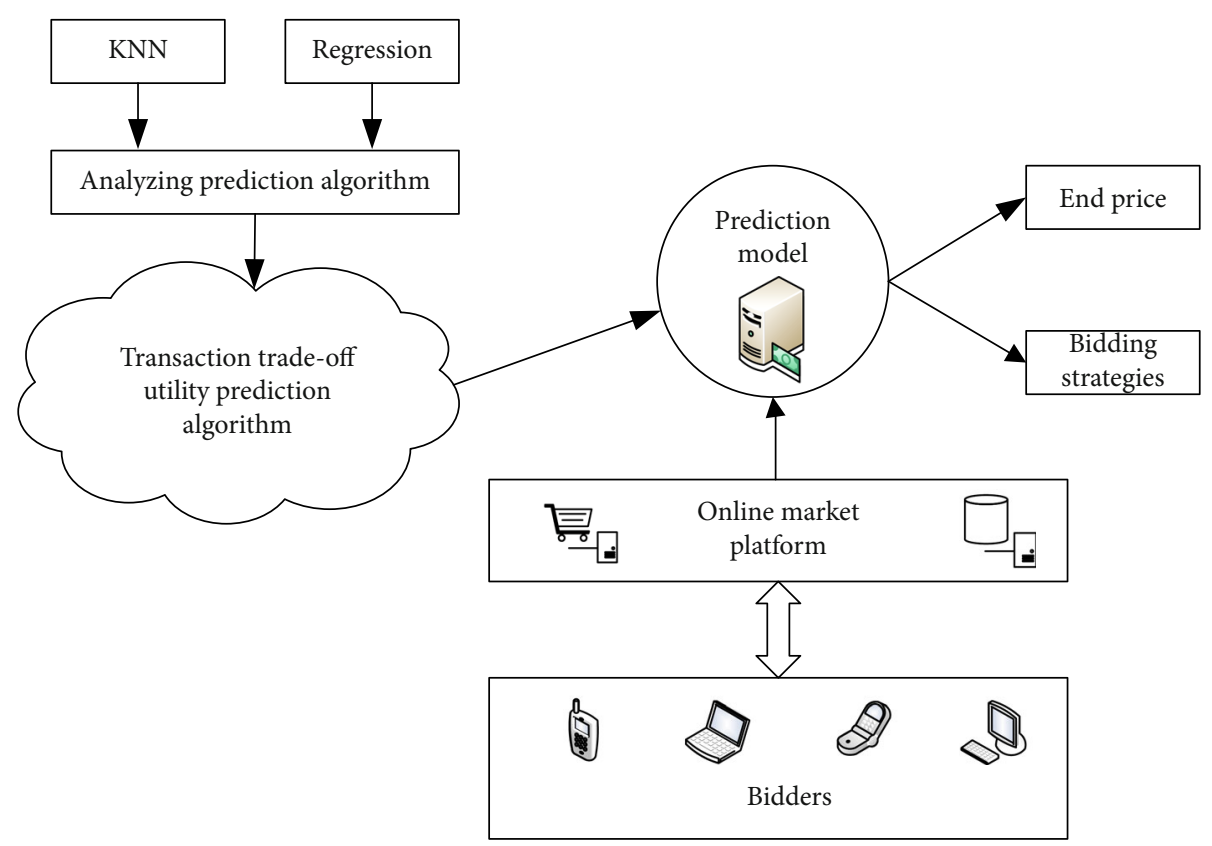

FIgURE 1: The architecture of an online auction system.

Transaction utility is considered as possibly the determinant that affects bidding behaviors [15]. In systems, the social welfare should be maximized through the design of incentive mechanism [16]. But many online auction formats including English auction, Dutch auction, first-price sealedbid, and second-price sealed-bid do not consider and calculate bidders' utility. Without considering bidders' utility and bidding motivation, the prediction algorithm with a good effect on a homogeneous dataset may not work well on heterogeneous datasets.

According to the above discussions, we research a transaction trade-off utility incentive mechanism and give the lemmas and proofs about item allocation problems in online auctions. In our model, the online auction framework of considering transaction utility is shown in Figure 1.

Agent technology is playing an increasingly important role in online auction platforms. An end-price dynamic forecasting agent (EDFA) is proposed, which can use the transaction trade-off utility incentive mechanism to predict whether an auction will be successful and how much end prices are in online auctions. Machine learning algorithms, which combine transaction trade-off utility, are used to predict final auction prices. EDFA predicts the end prices of online auctions in two phases: phase 1 for classifying online auctions by using the transaction trade-off utility function in $\mathrm{KNN}$ and phase 2 for predicting end prices of online auctions by regression. The results illustrate that the proposed algorithm considering utility not only improves the accuracy of a homogeneous dataset but also improves the accuracy of a heterogeneous dataset. As predicting whether an auction item will be sold, the proposed algorithm gave about $98 \%$ accuracy.

According to the bidding behaviors and price prediction problems in online auctions, the specific contributions of this work are shown as follows.
(1) To better understand the allocation process of auction items and transaction utility, we present a transaction trade-off utility incentive mechanism and the related lemmas and proofs. The proposed transaction trade-off utility incentive mechanism can maximize the utility of auction platforms and bidders

(2) Considering the transaction utility and bidding motivation, a transaction trade-off utility incentive mechanism is proposed. To improve the accuracy of classification and prediction, the transaction tradeoff utility function is proposed by combining KNN and regression named as the transaction trade-off utility prediction (TTUP) algorithm. The transaction trade-off utility function includes three aspects of GSP auctions, which are a reserve price, a clickthrough rate, and the number of item impressions. The function is used to classify in $\mathrm{KNN}$, and end prices of online auctions are predicted by regression

(3) We conduct comparison experiments on homogeneous and heterogeneous auction dataset to verify the effectiveness and accuracy based on the proposed transaction trade-off utility incentive mechanism and the TTUP algorithm. All results show that the proposed mechanism and algorithm are significantly better than other system algorithms both in terms of bidding behaviors and prediction accuracy

The rest of the paper is organized as follows. The related works are introduced in Section 2. In Section 3, we present the transaction trade-off utility incentive mechanism, including the proposed end-price dynamic forecasting agent, the system model, and the proposed algorithm TTUP. Experiments and results are explained in Section 4. We conclude the paper and provide our further research in Section 5. 
TABLE 1: The descriptions for notations in our incentive mechanism and algorithm.

\begin{tabular}{lc}
\hline Notation & Description \\
\hline$p$ & The reservation price of an online auction \\
$n$ & The impressions of auction items \\
$c$ & The click-through rate of auction items \\
$U$ & The bidder transaction trade-off utility as a \\
& function of relevant variables \\
\hline
\end{tabular}

\section{Related Works}

Bidders in online auctions face difficulties when looking for the best bidding strategies to win their interesting items. Many kinds of research focus on the design of bidding strategies. Kaur et al. [17] proposed a comprehensive methodology and designed bidding strategies with regression analysis and negotiation decision functions. Carbonneau and Vahidov [18] proposed an approach to facilitate multiattribute bidding in single-attribute auctions. Sayman and Akcay [19] indicated transaction utility can explain some bidding patterns on eBay. They showed that both underbidding and multiple bidding behaviors can be consistent with utility maximization if the buyer's utility incorporates a transaction utility component. Wang et al. [20-22] proposed a truthful incentive mechanism and improved the two-stage auction algorithm in mobile crowdsourcing. Efficient incentive mechanisms and auction algorithms can improve the efficiency and utility of the systems.

In the data mining and machine learning field, there are a lot of researches on predicting price. Many researchers used data mining techniques to predict price. The history auction data can be exploited for predicting the end-price of an auction by using support vector machines, $k$-nearest neighbor, clustering, regression, and multiple binary classifications [23-27]. Many different approaches have been proposed for predicting the end price of online auctions. Li et al. [28] used machine learning algorithms and traditional statistical methods to forecast the final prices of auction items. Ghani [29] predicted the end prices of online auctions using classification and regression trees, multiclass classification, and multiple binary classification methods. Heijst et al. [8] created a support system for predicting end prices on eBay using the CART regression tree. Khadge and Kulkarni [30] proposed a system using Naïve Bayes for classification and kernel mapping SVM for predicting whether an item maximizes profit or not. Moreover, if the model predicts the price of Nike shoes, a regression-type model will put equal weight on the shoe dataset, which may be inappropriate if the goal is to predict an auction price for a Sony laptop. While some of the brands and product differences can be controlled using appropriate predictor variables, there might still be intrinsic differences that are hard to measure. But we can measure the utility in different item transactions. As for the researches on using machine learning techniques and utility theory to predict the end price of the online auctions, fewer can be found.
The utility function is researched and adopted in some studies. Using utility function, which measures social welfare or satisfaction of a consumer as a function of consumption, can model different consumption behaviors [15]. In [31], the impact of time-based demand response programs on calculating incentive payments had been investigated considering the customer's utility function. In [32], the utility function was used to identify different customers' behavior and determine appropriate incentive payments to convince different customers to participate in the demand response program.

Logistic regression, Bayesian linear regression, decision trees, and deep recurrent neural network can be regarded as parametric models. Optimal parameters are usually different in different datasets, so the same group of parameters does not apply to predicting different item end-price of online auctions. The KNN method is a nonparametric model without strict assumption. However, there are many restrictions in the parametric models. To overcome these limitations of some parametric models, the proposed TTUP approach has better adaptability and robustness.

In generalized second-price (GSP) auctions, a reserve price is an important factor for a pricing model. The impact of a reserve price on GSP auctions was studied by Edelman and Schwarz [33]. In [33], the relationship between reserve prices and revenues was shown. Sellers want to have a relatively higher click-through rate (CTR) and a large number of impressions [34], which can increase their revenues. Thus, a reserve price, CTR, and the number of impressions were added to the proposed transaction trade-off utility function, and the function also follows this relationship in [33, 34].

Each bidder behaves independently based on his preferences. Few studies consider transaction utility in price forecasting. In this paper, we focus on identifying the bidding behavior of different bidders and predicting end prices considering the transaction trade-off utility function. We propose a novel trade-off utility approach for predicting online auction end prices based on the transaction trade-off utility incentive mechanism.

\section{The Proposed Transaction Trade-Off Utility Incentive Mechanism}

In this section, we mainly research the proposed transaction trade-off utility incentive mechanism, which includes the EDFA and the TTUP algorithm. We describe some attributes from the vast feature space of online auctions in Table 1.

3.1. The Proposed End-Price Dynamic Forecasting Agent. The EDFA is shown in Figure 2. The agent can use auction information to rank bidders and predict end prices of online auctions. Formally, our novel trade-off utility approach consists of four steps. Firstly, the bid server extracts auction history data and input it. Secondly, the utility-estimator and KNNestimator agent determines the best number $k$ of partitions for input data and then clusters the utility similar auctions together in $k$ groups. Thirdly, price-predictor forecasts end prices and designs bidding strategies by regression. Finally, the model is evaluated and deployed. Then, the optimized end prices and bidding strategies are output to the bid server. 


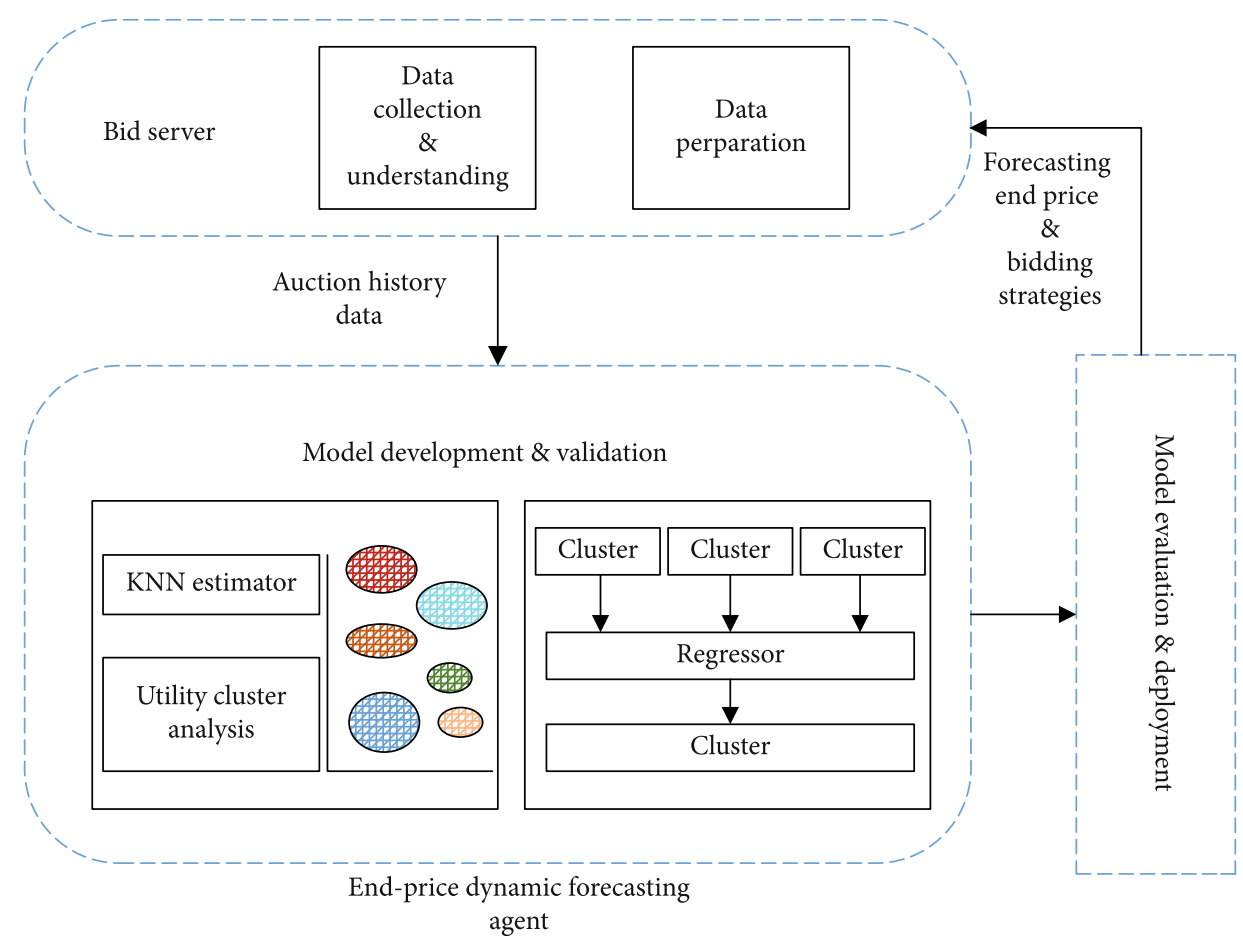

FIGURE 2: End-price dynamic forecasting agent.

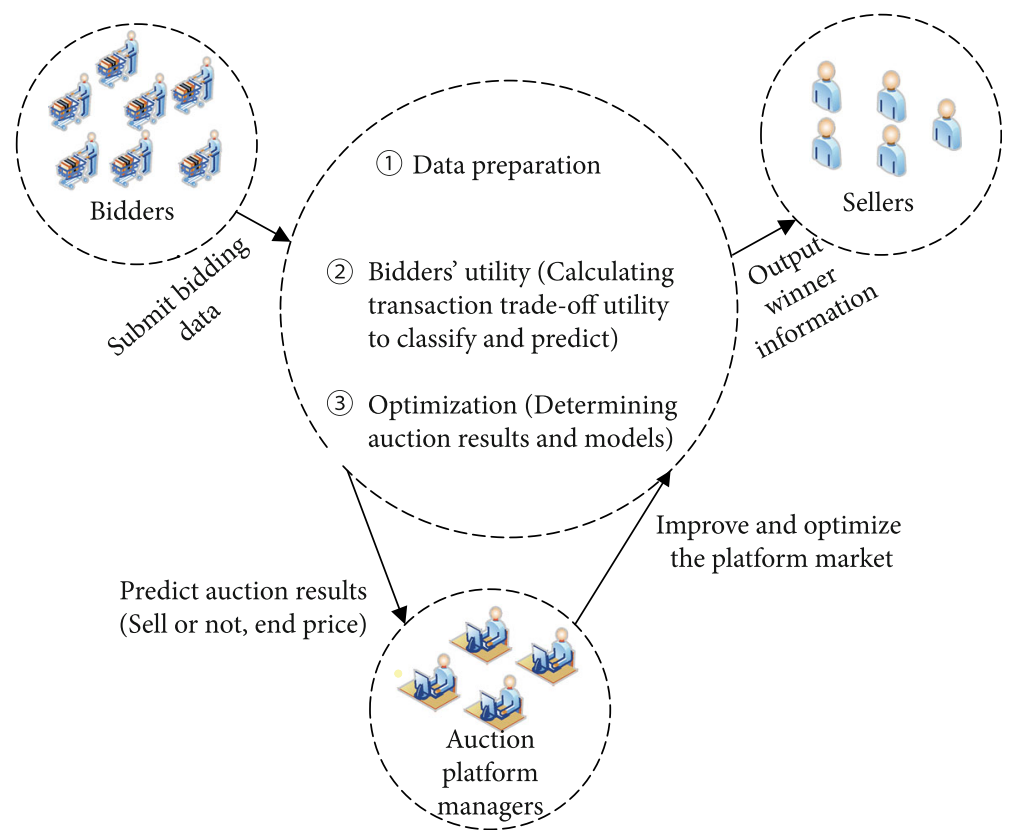

FIgURE 3: The process of the proposed incentive mechanism.

3.2. System Model. The utility is a form of measuring consumer satisfaction from commodity consumption and service. The utility function could accurately measure a consumer's preferences. As part of the process, factors such as customer satisfaction, total bid counts, and the rate of consumption by customers are considered key to accurately assessing the utility of the product. Unlike other forms of measuring the success of a given product, the utility function does not concern itself with the amount of return generated for the entity that manufactures and sells the product.

The transaction trade-off utility function is derived from a novel LP-based approach. It can be written as

$$
\psi(x)=1-\exp (x-1)
$$




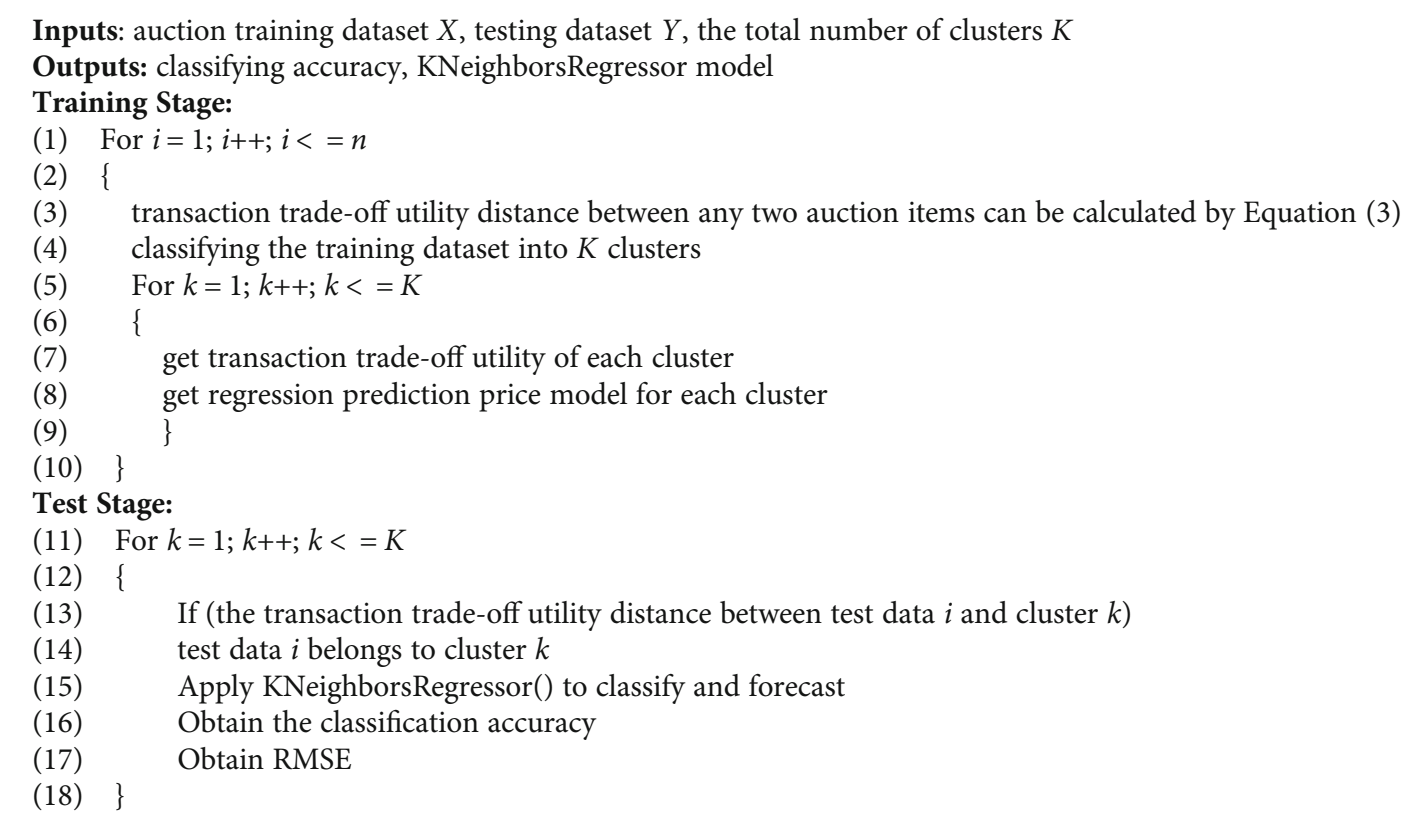

Algorithm 1: The proposed TTUP algorithm

Next, the $U$ value of each auction item will be calculated by Equation (2). We call $U$ as auction transaction trade-off utility. Let $U$ be the following function:

$$
U_{i}=c(i) \times \psi(f(i)),
$$

where $c(i)$ is the CTR of auction item $i ; f(i)$ is the fraction of a reserve price and auction item $i$ 's impression number, that is, $f(i)=p(i) / n(i)$, where $p(i)$ is a reserve price of an online auction for item $i$ and $n(i)$ is the number of auction item $i$ 's impressions.

Transaction trade-off utility distance is proposed to metric auction items. Suppose that auction item $i$ has $n$ feature variables $\left(x_{1}, x_{2}, \cdots, x_{n}\right)$, and the transaction trade-off utility of auction item $i$ is $U_{\mathrm{i}}$ calculated by Equation (2). Similarly, auction item $j$ also has $n$ feature variables $\left(y_{1}, y_{2}, \cdots, y_{n}\right)$, and the transaction trade-off utility of auction item $j$ is $U_{j}$ calculated by Equation (2). The transaction trade-off utility distance of item $i$ and item $j$ can be calculated as

$$
D_{i j}=\sqrt{\left(U_{i}-U_{j}\right)^{2} \sum_{i=1}^{n}\left(x_{i}-y_{i}\right)^{2}} .
$$

The process of the proposed incentive mechanism is shown in Figure 3. The core parts of the mechanism include data preparation, calculating transaction trade-off utility to classifying and predicting, and optimization.

3.3. The Proposed Transaction Trade-Off Utility Prediction Algorithm. In this section, we use the transaction trade-off utility distance metric to find $k$-nearest neighbors from auction items. An algorithm based on KNN can achieve a high
TABle 2: Data file description.

\begin{tabular}{lcc}
\hline Data file name & File description & Data rows \\
\hline TraingSet & $\begin{array}{c}\text { All auctions in April 2013 } \\
\text { All auctions in the first } \\
\text { TestSet }\end{array}$ & 258588 \\
TrainingSubset & $\begin{array}{c}\text { All auctions successfully } \\
\text { traded in April 2013 }\end{array}$ & 79760 \\
TestSubset & $\begin{array}{c}\text { All auctions successfully traded } \\
\text { in the first week of May 2013 }\end{array}$ & 9392 \\
\hline
\end{tabular}

level of accuracy in time series [35]. In [36], the utility had been modelled to determine the price.

Firstly, the transaction trade-off utility distance of the feature variables between the auction item $i$ and another auction item $j$ in the training dataset is calculated by Equation (3).

Secondly, all the auction items in the training set are sorted in ascending order according to the distance from item $j$.

Thirdly, $K$ data points with the smallest distance from item $i$ are select.

Finally, $K$ data points will be considered as the category of item $i$.

The proposed TTUP algorithm is described in Algorithm 1.

3.4. Properties of Proposed Transaction Trade-Off Utility Incentive Mechanism. With the emergence of new market and resource allocation models on the internet, there is a need for a new artificial intelligence algorithmic theory of combining utility theory and machine learning algorithms. We call bidders with very simple utility functions "single- 
minded bidders" [37]. The proposed algorithm can help understand online auction repercussions to bid price, auction strategies, bidding behaviors, and social welfare caused by auction mechanisms or transaction utility.

Considering that an online auction website is composed of a set $N=\{1,2, \cdots, n\}$ of items and a set $M=\{1,2, \cdots, m\}$ of bidders. For each bidder $i$, if he bids for item $j$, he will get the utility $U_{i j}$ and pay $P_{i j}$ for bidding item $j$. In the online auction platform, the objective function of each bidder is shown as follows:

$$
\begin{aligned}
& \max \sum_{i=1}^{m} \sum_{j \in N} U_{i j}, \\
& \text { s.t. } \cdot \sum_{j \in N} P_{i j} \leq B_{i} \cdot \forall i \in M,
\end{aligned}
$$

where $B_{i}$ is the possessed budget by bidder $i$.

We assume that the customers, who bid for the same quantity of items, have the same utility and the same bidding price. In online auctions, there are different reserved prices, different bidding strategies, and different budgets. A uniform price on all items is not feasible, so each bidder will not necessarily get items that she is interested in. We will find that not all items can be sold at a uniform bidding price.

In the book of algorithmic game theory [37], the combinatorial auction problem statement is introduced by Blumrosen and Nisan. As they introduced the transaction utility, we have the following definitions by the proposed transaction utility.

Definition 1. A utility $u$ is a real-utility function that for each subset $S$ of items, $u(S)$ is the total utility that bidder $i$ obtains if he receives this bundle of bidding items.

Definition 2. An allocation of the bidding items among the bidders is $S_{1}, \cdots, S_{n}$, where $S_{i} \cap S_{j}=\varnothing$ for every $i \neq j$. The total utility obtained by an allocation is $\sum_{i} u_{i}\left(S_{i}\right)$. A socially efficient allocation (among bidders with utility valuations $u_{1}$, $\cdots, u_{n}$ ) is an allocation with maximum social welfare and utility among all allocations.

Definition 3. The allocation problem among single-minded bidders is the following:

Input: $\left(S_{\mathrm{i}}{ }^{*}, u_{\mathrm{i}}{ }^{*}\right)$ for each bidder $i=1, \cdots, n$, where $S_{\mathrm{i}}{ }^{*}$ is a bundle of bidding items and $u_{\mathrm{i}}{ }^{*}$ is a utility valuation.

Output: a subset of winning bids $W \subseteq\{1, \cdots, n\}$ such that for every $i \neq j \in W, S_{i}{ }^{*} \cap S_{j}{ }^{*}=\varnothing$ with maximum social welfare $\sum_{i \in W} u_{i}{ }^{*}$.

Lemma 4. The proposed transaction trade-off utility incentive mechanism is computationally efficient.

Proof. In the proposed transaction trade-off utility incentive mechanism, KNN and regression algorithms are applied to bidder grouping and price forecasting. When the number of samples is $n$, the time complexity is $O(n)$ in the KNN algorithm. Besides, when samples are divided into $k$ clusters,

\begin{tabular}{|c|c|}
\hline Feature name & Feature description \\
\hline Price & End prices of auctions \\
\hline StartingBid & $\begin{array}{c}\text { Minimum transaction price of an } \\
\text { auction }\end{array}$ \\
\hline BidCount & Number of bids won in an auction \\
\hline Title & Transaction title \\
\hline QuantitySold & Successful sale number ( 0 or 1$)$ \\
\hline SellerRating & Seller's rating on eBay \\
\hline StartDate & Auction start date \\
\hline EndDate & Auction end date \\
\hline PositiveFeedbackPercent & $\begin{array}{l}\text { Percentage of positive feedback } \\
\text { received by seller (for all } \\
\text { feedback) }\end{array}$ \\
\hline BuyitNowPrice & Price for immediate purchase \\
\hline HighBidderFeedbackRating & $\begin{array}{c}\text { eBay rating of the highest-price } \\
\text { bidder }\end{array}$ \\
\hline IsHOF & $\begin{array}{l}\text { The seller is or not a hall of fame } \\
\text { player }(0 \text { or } 1)\end{array}$ \\
\hline AvgPrice & $\begin{array}{l}\text { Average price of a good in } \\
\text { inventory }\end{array}$ \\
\hline MedianPrice & $\begin{array}{l}\text { Median price of a good in } \\
\text { inventory }\end{array}$ \\
\hline AuctionCount & $\begin{array}{c}\text { Total number of auctions in } \\
\text { inventory }\end{array}$ \\
\hline SellerSaleToAveragePriceRatio & $\begin{array}{l}\text { Proportion of auction goods price } \\
\text { to average price }\end{array}$ \\
\hline StartDayOfWeek & $\begin{array}{l}\text { The beginning day of the auction } \\
\text { in a week }\end{array}$ \\
\hline EndDayOfWeek & $\begin{array}{l}\text { The end day of the auction in a } \\
\text { week }\end{array}$ \\
\hline AuctionDuration & Auction duration days \\
\hline StartingBidPercent & $\begin{array}{c}\text { The ratio of the starting bidding } \\
\text { price to the average transaction } \\
\text { price }\end{array}$ \\
\hline SellerClosePercent & $\begin{array}{c}\text { The proportion of a seller's } \\
\text { successful auctions to all online } \\
\text { auctions }\end{array}$ \\
\hline ItemAuctionSellPercent & $\begin{array}{l}\text { Percentage of successful auctions } \\
\text { in all online auctions }\end{array}$ \\
\hline
\end{tabular}

TABLe 3: The main features and descriptions of dataset 1.

the prediction price time complexity is $O(n * k)$ in the TTUP algorithm. The proposed transaction trade-off utility incentive mechanism is computationally efficient because the bidding items and bidders can be selected in polynomial time.

Lemma 5. The proposed transaction trade-off utility incentive mechanism is truthful.

Proof. When classifying the bidders into $K$ clusters by transaction trade-off utility distance, the TTUP algorithm considers reservation price, the total bid counts of an auction item, and the creditability of a bidder. In online auctions, each bidder wants to maximize total utility, which indicates that bidders should tell their truthfulness. Therefore, the 

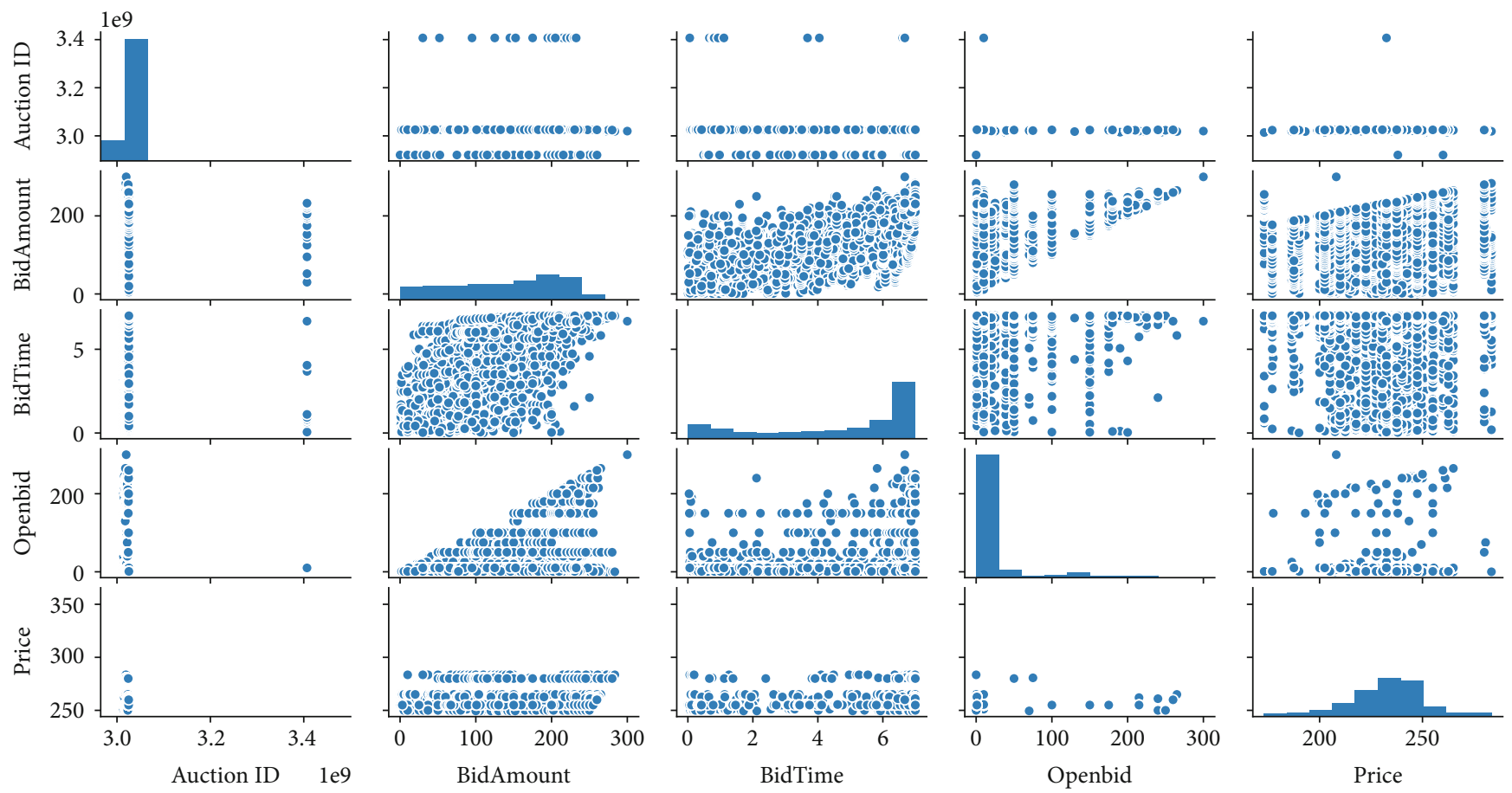

FIGURE 4: The illustration of impact from bid characteristics.

proposed transaction trade-off utility incentive mechanism is truthful.

Lemma 6. The proposed transaction trade-off utility incentive mechanism can maximize social welfare.

Proof. In the proposed transaction trade-off utility incentive mechanism, social welfare can be shown by $\sum_{i \in W} u_{i}{ }^{*}$, where $W \subseteq\{1, \cdots, n\}$ is a subset of winning bids. Therefore, social welfare can be maximized based on the utility of bidders. It indicates that the proposed transaction trade-off utility incentive mechanism can maximize the social welfare of online auction platforms.

\section{Experiment and Result Analysis}

\subsection{Evaluation Metrics}

4.1.1. Discrete Prediction. When we predict an auction item will sell or not, it is a classification problem. We can use an accuracy metric to judge the performance of our algorithm. Accuracy metric is defined as follows:

$$
\text { accuracy }=\frac{\mathrm{TC}}{\mathrm{TN}} \times 100 \% \text {, }
$$

where TC is the number of correct prediction samples and $\mathrm{TN}$ is the total number of prediction samples.

4.1.2. Continuous Prediction. When we predict the end prices of online auctions, it is a continuous problem. We can use the root mean square error (RMSE) to evaluate the prediction performance. RMSE is a widely used numerical prediction evaluation index. It measures the average deviation degree
TABLE 4: Trade-off utility values with different parameters.

\begin{tabular}{lcccc}
\hline Item & $c(i)$ & $p(i)$ & $n(i)$ & $\begin{array}{c}U_{i}=c(i) \times \psi(f(i)) \\
f(i)=p(i) / n(i)\end{array}$ \\
\hline A & 0.1 & 0.1 & 100 & 0.06318 \\
B & 0.1 & 0.3 & 100 & 0.06310 \\
C & 0.1 & 0.5 & 100 & 0.06303 \\
D & 0.1 & 0.8 & 100 & 0.06292 \\
E & 0.1 & 1 & 100 & 0.06284 \\
F & 0.3 & 0.1 & 100 & 0.18953 \\
G & 0.3 & 0.3 & 100 & 0.18930 \\
H & 0.5 & 0.1 & 500 & 0.31602 \\
I & 1 & 0.1 & 500 & 0.63205 \\
\hline
\end{tabular}

between the predicted values and the actual values. The smaller the value of RMSE is, the better it is. RMSE is defined as follows:

$$
\mathrm{RMSE}=\sqrt{\mathrm{MSE}}=\sqrt{\frac{1}{n} \sum_{i=1}^{n} E_{i}^{2}}=\sqrt{\frac{1}{n} \sum_{i=1}^{n}\left(y_{i}-y \wedge_{i}\right)^{2}},
$$

where $y_{i}$ is the actual value of sample $i, \widehat{y}_{i}$ is the estimate of sample $i$, and $n$ is the total number of samples.

4.2. Data. In this section, we use two datasets with eBay auctions. Dataset 1 is downloaded from https://cims.nyu.edu/ $\sim$ munoz/data/. The dataset contains four data files that are described in Table 2. Dataset 2 is a real-world dataset on Canon that we used a special collection program to collect from eBay. The dataset contains 4889 auction data rows. 


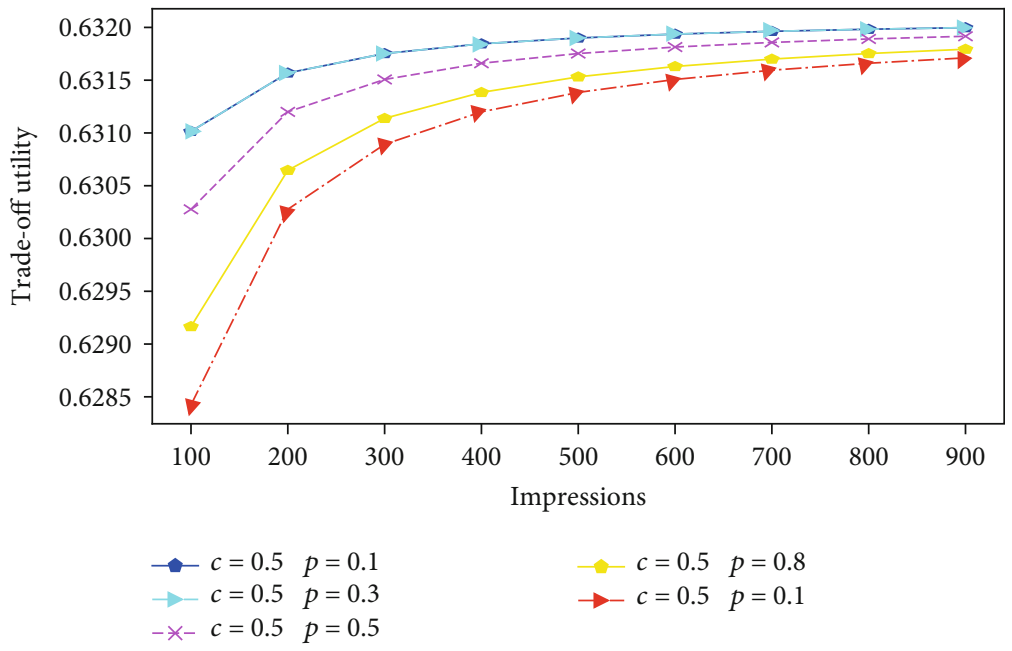

FIgURE 5: The trade-off utility as a function of impressions.

TABLE 5: Model performance.

\begin{tabular}{lcccc}
\hline & \multicolumn{2}{c}{ Dataset 1 } & \multicolumn{2}{c}{ Dataset 2 } \\
& Accuracy & RMSE & Accuracy & RMSE \\
\hline TTUP & $98.45 \%$ & 4.56 & $97.52 \%$ & 5.21 \\
KNN & $86.53 \%$ & 5.11 & $88.56 \%$ & 7.96 \\
Linear regression & $82.67 \%$ & 5.56 & $87.67 \%$ & 8.79 \\
CART (regression tree) & $94.72 \%$ & 4.88 & $95.33 \%$ & 6.16 \\
SVM & $95.74 \%$ & 4.97 & $94.28 \%$ & 6.20 \\
\hline
\end{tabular}

We use $70 \%$ of the dataset as the training data and $30 \%$ of the dataset as the test set.

The main feature names and descriptions of dataset 1 are shown in Table 3 . Independent variable analysis is the main diagnostic process used to obtain reliable prediction results. Because there are many bid characteristics of online auction data, it is essential to analyze the relationship and distribution of the independent variables before modelling. Some main characteristics, which are related to auction price, could be found by bid characteristic analysis. Figure 4 is a scatter matrix of auction characteristics in dataset 2 , which illustrates the impact of bid characteristics. The diagonal is the histogram of characteristic variables. Through the histogram, we can see that the price histogram illustrates that price obeys normal distribution.

4.3. Numerical Simulation and Analysis. Table 4 shows the calculated trade-off utility values with different online auction parameters. This has a bigger trade-off utility value in the relatively higher range of CTR.

Figure 5 represents the trade-off utilities as a function of impressions. With these online auction parameters, the lower the reserve prices are, the more the trade-off utilities are at the same CTR, and the number of impressions. As the reserve prices increase, the trade-off utilities fall. However, if the number of impressions exceeds certain values, the reduction will be less sharp. When the number of impressions reaches a certain number, the utilities tend to converge.
Table 5 shows accuracy and RMSE in the existing system and proposed system. The results demonstrate that the endprice dynamic forecasting agent who adopts the transaction trade-off utility approach outperforms agents following other methodologies. The proposed system using the TTUP algorithm gives $98.45 \%$ and $97.52 \%$ accuracy. The proposed system performs best compared with other algorithms. It also can be found that transaction trade-off utility is a potential driver of bidders' behaviors in bidding. Transaction tradeoff utility is also an important factor for predicting end prices in online auctions.

\section{Conclusions}

In this paper, we present a transaction trade-off utility incentive mechanism and the related lemmas and proofs. The proposed EDFA is based on the incentive mechanism and system model. The contribution of this study is twofold: it is the first study that proposes the transaction trade-off utility incentive mechanism and transaction trade-off utility function, and it is the first study that uses transaction utility in the prediction of online auction end prices. Considering the transaction utility, our system is good for bidders, sellers, and the platform markets. Furthermore, social welfare is also maximized. We tested our price prediction model in a series of experiments. For both homogeneous and heterogeneous datasets, our model gives better accuracy. This proposed transaction trade-off utility incentive mechanism can be used in other auction prediction systems. Building the EDFA is then started automatically.

In further work, we plan to use our transaction utility incentive mechanism in reinforcement learning and transfer learning. Besides, we will combine offline with online data to predict the end prices of online auctions.

\section{Data Availability}

Dataset 1 in this study can be downloaded from https://cims .nyu.edu/ munoz/data/. Dataset 2 is available upon request from the first author. 


\section{Conflicts of Interest}

The authors declare that they have no conflicts of interest regarding the publication of this paper.

\section{Acknowledgments}

We acknowledge the support from the National Science Foundation of China (No. 61472095). This work is partially supported by the Natural Science Foundation of Heilongjiang Province under Grant No. LH2020F023, and by the Educational Science Planning of Heilongjiang Province under Grant No. ZJB1421113 and No. GJB1421252.

\section{References}

[1] K. Hasker and R. Sickles, "eBay in the economic literature: analysis of an auction marketplace," Review of Industrial Organization, vol. 37, no. 1, pp. 3-42, 2010.

[2] Y. Wang, Y. Gao, Y. Li, and X. Tong, "A worker-selection incentive mechanism for optimizing platform-centric mobile crowdsourcing systems," Computer Networks, vol. 171, article 107144, 2020.

[3] E. Haruvy and P. T. L. P. Leszczyc, "Internet auctions," Foundations and Trends( $R)$ in Marketing, vol. 4, no. 4, pp. 1-75, 2010.

[4] Z. Cai, S. Ji, J. He, L. Wei, and A. G. Bourgeois, "Distributed and asynchronous data collection in cognitive radio networks with fairness consideration," IEEE Transactions on Parallel and Distributed Systems, vol. 25, no. 8, pp. 2020-2029, 2014.

[5] V. Nikolaidou and P. A. Mitkas, "A sequence mining method to predict the bidding strategy of trading agents," in Agents and Data Mining Interaction. ADMI 2009. Lecture Notes in Computer Science, vol 5680, pp. 139-151, Springer, Berlin, Heidelberg, 2009.

[6] Z. Cai and X. Zheng, "A private and efficient mechanism for data uploading in smart cyber-physical systems," IEEE Transactions on Network Science and Engineering, vol. 7, no. 2, pp. 766-775, 2020.

[7] Z. Cai, Z. He, X. Guan, and Y. Li, "Collective data-sanitization for preventing sensitive information inference attacks in social networks," IEEE Transactions on Dependable and Secure Computing, vol. 15, no. 4, pp. 577-590, 2018.

[8] D. V. Heijst, R. Potharst, and M. V. Wezel, "A support system for predicting eBay end prices," Decision Support System, vol. 44, pp. 970-982, 2008.

[9] M. Bin and M. Kamel, "Intelligent system for price premium prediction in online auctions," International Journal of Advanced Computer Science and Applications, vol. 11, no. 3, pp. 329-334, 2020.

[10] S. Zhang, W. Jank, and G. Shmueli, "Real-time forecasting of online auctions via functional K-nearest neighbors," SSRN Electronic Journal, vol. 26, pp. 666-683, 2010.

[11] Z. He, Z. Cai, J. Yu, X. Wang, Y. Sun, and Y. Li, "Cost-efficient strategies for restraining rumor spreading in mobile social networks," IEEE Transactions on Vehicular Technology, vol. 66, no. 3, pp. 2789-2800, 2017.

[12] A. L. Jin, W. Song, and W. Zhuang, "Auction-based resource allocation for sharing cloudlets in mobile cloud computing," IEEE Transactions on Emerging Topics in Computing, vol. 6, no. 1 , pp. $45-57,2018$.
[13] W. Shi, L. Zhang, C. Wu, Z. Li, and F. C. M. Lau, "An online auction framework for dynamic resource provisioning in cloud computing," IEEE/ACM Transactions on Networking, vol. 24, no. 4, pp. 2060-2073, 2016.

[14] Y. Wang, Z. Cai, Z.-H. Zhan, B. Zhao, X. Tong, and L. Qi, "Walrasian equilibrium-based multiobjective optimization for task allocation in mobile crowdsourcing," IEEE Transactions on Computational Social Systems, vol. 7, no. 4, pp. 1033-1046, 2020.

[15] C. Millan, "Theory of utility and consumer behaviour: a comprehensive review of concepts, properties and the most significant theorems," in Utility and Production. Contributions to Economics, Physica, Heidelberg, 1999.

[16] Y. Wang, Z. Cai, Z.-H. Zhan, B. Zhao, X. Tong, and L. Qi, “An optimization and auction-based incentive mechanism to maximize social welfare for mobile crowdsourcing," IEEE Transactions on Computational Social Systems, vol. 6, no. 3, pp. 414429, 2019.

[17] P. Kaur, M. Goyal, and J. Lu, “A comparison of bidding strategies for online auctions using fuzzy reasoning and negotiation decision functions," IEEE Transactions on Fuzzy Systems, vol. 25, no. 2, pp. 425-438, 2017.

[18] R. Carbonneau and R. Vahidov, "A multi-attribute bidding strategy for a single-attribute auction marketplace," Expert Systems with Applications, vol. 43, no. 1, pp. 42-50, 2016.

[19] S. Sayman and Y. Akcay, "A transaction utility approach for bidding in second-price auctions," Journal of Interactive Marketing, vol. 49, no. 2, pp. 86-93, 2020.

[20] Y. Wang, Z. Cai, X. Tong, Y. Gao, and G. Yin, "Truthful incentive mechanism with location privacy-preserving for mobile crowdsourcing systems," Computer Networks, vol. 135, pp. 32-43, 2018.

[21] Y. Wang, Z. Cai, G. Yin, Y. Gao, X. Tong, and G. Wu, “An incentive mechanism with privacy protection in mobile crowdsourcing systems," Computer Networks, vol. 102, pp. 157-171, 2016.

[22] Y. Wang, G. Yin, Z. Cai, Y. Dong, and H. Dong, "A trust-based probabilistic recommendation model for social networks," Journal of Network and Computer Applications, vol. 55, pp. 59-67, 2015.

[23] I. Raykhel and D. Ventura, "Real-time automatic price prediction for eBay online trading," in Proceedings of the TwentyFirst Conference on Innovative Applications of Artificial Intelligence, Pasadena, CA, USA, July 2009.

[24] S. Khwaja, M. Naeem, A. Anpalagan, A. Venetsanopoulos, and B. Venkatesh, "Improved short-term load forecasting using bagged neural networks," Electric Power Systems Research, vol. 125, pp. 109-115, 2015.

[25] L. Yu, Z. Wang, and L. Tang, “A decomposition-ensemble model with data-characteristic-driven reconstruction for crude oil price forecasting," Applied Energy, vol. 156, pp. 251-267, 2015.

[26] J. Mendes-Moreira, C. Soares, A. M. Jorge, and J. F. D. Sousa, "Ensemble approaches for regression," ACM Computing Surveys, vol. 45, no. 1, pp. 1-40, 2012.

[27] M. Oliveira and L. Torgo, "Ensembles for time series forecasting," Proceedings of Asian Conference on Machine Learning, vol. 39, pp. 360-370, 2014.

[28] X. Li, L. Liu, L. Wu, and Z. Zhang, "Predicting the final price of online auction items," Expert Systems with Application, vol. 31, no. 3, pp. 542-550, 2006. 
[29] R. Ghani, "Price prediction and insurance for online auctions," KDD '05: Proceedings of the eleventh ACM SIGKDD international conference on Knowledge discovery in data mining, 2005, pp. 411-418, New York, NY, USA, August 2005.

[30] M. R. Khadge and M. V. Kulkarni, "Machine learning approach for predicting end price of online auction," in 2016 International Conference on Inventive Computation Technologies (ICICT), Coimbatore, India, August 2017.

[31] J. Wang and B. Ravindran, "Time-utility function-driven switched Ethernet: packet scheduling algorithm, implementation, and feasibility analysis," IEEE Transactions on Parallel and Distributed Systems, vol. 15, no. 2, pp. 119-133, 2004.

[32] A. Niromandfam, A. S. Yazdankhah, and R. Kazemzadeh, "Modeling demand response based on utility function considering wind profit maximization in the day-ahead market," Journal of Cleaner Production, vol. 251, article 119317, 2019.

[33] B. Edelman and M. Schwarz, Optimal auction design in a multi-unit environment: the case of sponsored search auctions, working paper, Harvard University, 2006.

[34] K. Ren, W. Zhang, K. Chang, Y. Rong, Y. Yu, and J. Wang, "Bidding machine: learning to bid for directly optimizing profits in display advertising," IEEE Transactions on Knowledge \& Data Engineering, vol. 30, no. 4, pp. 645-659, 2018.

[35] D. Xu, Y. Wang, P. Peng, S. Beilun, Z. Deng, and H. Guo, "Real-time road traffic state prediction based on kernelKNN," Transportmetrica A: Transport Science, vol. 16, no. 1, pp. 104-118, 2020.

[36] N. Cicek and H. Delic, "Demand response management for smart grids with wind power," IEEE Transactions on Sustainable Energy, vol. 6, no. 2, pp. 625-634, 2015.

[37] N. Nisan, T. Roughgarden, E. Tardos, and V. Vazirani, Algorithmic Game Theory, Cambridge University Press, Cambridge, 2020. 